\title{
Video Article \\ Measurement of Carotenoids in Perifovea using the Macular Pigment Reflectometer
}

\author{
Juan C. Sanabria ${ }^{1}$, Jordan Bass ${ }^{1}$, Frank Spors ${ }^{1}$, Dennis L. Gierhart ${ }^{2}$, Pinakin Gunvant Davey ${ }^{1}$ \\ ${ }^{1}$ College of Optometry, Western University of Health Sciences \\ ${ }^{2}$ ZeaVision LLC
}

Correspondence to: Pinakin Gunvant Davey at contact@pinakin-gunvant.com

URL: https://www.jove.com/video/60429

DOI: doi: $10.3791 / 60429$

Keywords: Medicine, Issue 155, macular pigment reflectometry, heterochromatic flicker photometer, lutein, zeaxanthin, retina, macular degeneration, macular pigment optical density

Date Published: 1/29/2020

Citation: Sanabria, J.C., Bass, J., Spors, F., Gierhart, D.L., Davey, P.G. Measurement of Carotenoids in Perifovea using the Macular Pigment Reflectometer. J. Vis. Exp. (155), e60429, doi:10.3791/60429 (2020).

\section{Abstract}

The macular pigment reflectometer (MPR) objectively measures the overall macular pigment optical density (MPOD) and further provides the lutein optical density (L-OD) and zeaxanthin optical density (Z-OD) in the central 1 degree of the fovea. A modification of the technique was developed to evaluate in vivo carotenoid density eccentric to the fovea. An adjustable track system with red LED lights was placed $6.1 \mathrm{~m}$ away from the participant to facilitate ocular fixation. Lights were spaced appropriately to create increments of 1 degree retinal disparity during the reflectometry measurements. All reflectometry measurements were obtained with pupillary dilation. The mean MPR-MPOD value for the central measurement was 0.593 (SD 0.161) with an L-OD to Z-OD ratio of 1:2.61. The MPR-MPOD value at 1 degree was 0.248 and the mean MPRMPOD value at 2 degrees in the parafoveal region was 0.143 . The L-OD to Z-OD ratio at 1 degree and 2 degrees off center was $1.38: 1.0$ and 2.08:1.0, respectively. The results demonstrate that MPOD measurements obtained using the MPR decrease as a function of retinal eccentricity and that there is a higher concentration of zeaxanthin centrally compared to lutein. The L-OD to Z-OD ratio changes with foveal eccentricity, with twice more lutein than zeaxanthin at 2 degrees off center. Our technique successfully provides a quick in vivo method for the measurement of macular pigment optical density at various foveal eccentricities. The results agree with prior published in vivo and in vitro xanthophyll carotenoid density distribution measurements.

\section{Video Link}

The video component of this article can be found at https://www.jove.com/video/60429/

\section{Introduction}

Age related macular degeneration (AMD) is a leading cause of blindness and accounts for $8.7 \%$ of blindness worldwide ${ }^{1}$. Risk factors associated with AMD include increasing age, female gender, smoking, light iris color, lipid imbalance, lifetime exposure to sunlight and ultraviolet radiations, systemically lower levels of antioxidants, lower macular pigment optical density (MPOD), genetics, and race ${ }^{2}$. Of these, modifiable risk factors are smoking cessation, oral supplementation of antioxidants, and carotenoids. Carotenoids are natural pigments found in plants and microorganisms, and are efficient antioxidants ${ }^{3}$. They are produced by photosynthetic organisms; humans obtain carotenoids from their diet ${ }^{3,4}$. Macular pigments are composed of three carotenoids: lutein, zeaxanthin, and meso-zeaxanthin ${ }^{4}$. The xanthophylls lutein and zeaxanthin ${ }^{5}$ are found in the retina, specifically the macula, and give the fovea its yellow color ${ }^{6}$. Higher concentrations of xanthophylls are observed in the axons of the photoreceptors and inner plexiform layers of the retina ${ }^{5,7}$. The intake of carotenoids, like lutein and zeaxanthin, increases the level of macular pigment. Lutein and zeaxanthin are obtained from dietary intake or with nutrient supplementation, while meso-zeaxanthin is simply a byproduct of the metabolism of lutein ${ }^{3,7,8}$. Lutein and zeaxanthin concentrations differ in the various regions of the retina. Centrally, in the fovea, zeaxanthin concentration is greater than that of lutein, with a ratio of $2.3: 1^{1,10}$. The concentration of carotenoids decreases 100 -fold per mm in the foveal periphery, where lutein is more prevalent than zeaxanthin, with a ratio of $2.4: 1^{9,10}$.

The presence of xanthophylls in the retina protects the retinal circuitry, especially in the fovea and macula, and is critical for central vision. The xanthophylls protect the retina by two possible mechanisms: 1) filtering blue light and 2) decreasing oxidative stress ${ }^{5,11,12,13}$. Blue light scatters the most in the retina and higher levels of macular pigment centrally absorb the scattered light, thereby improving vision. Additionally, the blue part of the visible spectrum is composed of high energy, short wavelengths that can result in the production of excessive amounts of reactive oxygen species in the retina. Therefore, it is thought that carotenoids reduce the oxidative burden on the macula by acting as antioxidants in the inner retina and photoreceptor retinal pigment epithelial complex by quenching these free radicals ${ }^{5,12,13,14}$.

Measurement of retinal carotenoids has larger implications in systemic health. A recent trial showed that carotenoid therapy improves retinal function in diabetics without any alterations to blood glucose levels ${ }^{15}$. The levels of carotenoid density in the retina are also strongly correlated with the levels in the brain ${ }^{16}$. Carotenoid levels may be crucial in the developmental years ${ }^{17,18}$, and levels in the brain decline with age $^{19}$. The MPOD levels are related to neuroprotection and neural efficiency in both children and the elderly ${ }^{20,21}$. Thus, there is a need to 
measure MPOD and its characteristics clinically. This will play a role in diagnosis, management, and treatment of various ocular and systemic conditions $7,15,16,17,18,19,20,21$.

The current commercially available MPOD measuring technologies are heterochromatic flicker photometers (HFP), which are based on psychophysical testing. These measure a 1 degree patch on the fovea, which amounts to a $\sim 0.30 \mathrm{~mm}$ diameter circle ${ }^{22}$. While these types of devices have been shown to be reliable, they are limited by their subjective nature, are time consuming to use, and are unable to distinguish the individual quantities of xanthophylls that form MPOD ${ }^{13,22,23,24}$. The macular pigment reflectometer (see Table of Materials), also referred to as a reflectometer (see Figure 1), addresses these limitations by objectively measuring the MPOD and its individual components of lutein and zeaxanthin (xanthophylls) ${ }^{25}$. The reflectometer utilizes a UV/IR filtered and collimated quartz halogen source to send a controlled light beam to the retina (see schematic Figure 2) and the internal filters absorb most of the radiation produced. Therefore, there is little to no risk of radiation exposure for the participant. The various chromophores and structures in the human eye and the corresponding absorption and reflectance patterns are well described in the literature ${ }^{26,27,28}$. Analysis of the reflected light processed by the internal spectrometer allows for the quantitative isolation and measurement of lutein and zeaxanthin optical densities (L-OD, Z-OD) along with overall MPOD. The third retinal carotenoid mesozeaxanthin is spectrally indistinguishable from zeaxanthin and thus the Z-OD represents a combination of both carotenoids ${ }^{29}$. Prior work has shown reflectometry to be reliable when measuring central L-OD, Z-OD, and MPOD ${ }^{25,29}$.

The purpose of the current study is to create a technique that can be utilized to produce in vivo estimates of zeaxanthin and lutein levels in the foveal and parafoveal retinal regions in humans. Additional aims are to compare the findings to previously published laboratory and histology results $^{14,29}$. The approach developed and described in this manuscript and its utilization alongside reflectometry to measure the perifoveal MPOD is novel. This technique can be used with any existing reflectometry unit without major modification to measure retinal levels of individual carotenoids, such as L-OD and Z-OD, at various foveal and parafoveal locations.

The study presented in this manuscript includes eight participants ranging from 22-29 years of age. Our methods include first conducting a routine ophthalmic examination to ensure that the study participants meet the inclusion criteria. After obtaining informed consent, each study participant underwent the following four tests: 1) a commercially available heterochromatic flicker photometer device was utilized to obtain a central MPOD measurement; 2) a reflectometer device was utilized to obtain two central measurements; 3) using the same reflectometer device in conjunction with the peripheral track system, measurements of carotenoid levels at a 1 degree eccentricity, that is a $0.30 \mathrm{~mm}$ diameter circle, was centered at $0.30 \mathrm{~mm}$ from the central fovea; 4) using the same set-up, carotenoid levels at a 2 degree eccentricity, a $0.30 \mathrm{~mm}$ diameter circle placed at the edge of the fovea (a parafoveal region), were also measured.

The MPR measurements were performed after dilating each participant's pupil with $1 \%$ tropicamide ophthalmic drops. It is known that pupillary dilation is not needed to obtain MPOD values using reflectometry, but it may improve the repeatability of L-OD and Z-OD measurements ${ }^{25,29}$. This is possibly due to the fact that measurements obtained from the retina using the reflectometer had better signal-to-noise ratio when the pupils were dilated. For the accurate and stable peripheral reflectometry measurements, participants used fixation targets that were placed at optical infinity ${ }^{30,31}$.

We obtained reflectometer measurements for $30 \mathrm{~s}$ and discarded the first $10 \mathrm{~s}$ of data. This procedure has two advantages: 1) the signal source is bright and allows for the eyes to adapt and adjust to the task; and 2) most importantly, the photoreceptor pigment bleaches during the first 10 $\mathrm{s}$. Therefore, eliminating the first $10 \mathrm{~s}$ of measurement allows for a more stable and accurate signal ${ }^{29}$. We performed all reflectometry tests twice in the present study, after which we averaged the measurements to obtain mean MPOD, L-OD, and Z-OD values and the ratio of Z-OD/ L-OD for each participant.

\section{Protocol}

All subjects were recruited at a single site, the Western University of Health Sciences. The study was approved by the institutional review board at Western University of Health Sciences, Pomona, California, USA, and conducted in accordance with the tenets of the Declaration of Helsinki. Prior to participation all participants were given a detailed explanation of the study and signed an informed consent form before any standard ophthalmic evaluation was carried out.

\section{Participant recruitment}

1. Include participants that are at least 18 years of age and have a visual acuity of $20 / 40$ or better.

2. Include participants with clinically insignificant conditions like cataracts, isolated drusen, posterior vitreous detachment, familial drusen in periphery, and peripheral retinal conditions, such as lattice degeneration, or retinal pigment epithelial defects. Ensure that the participants have normal binocularity and that they do not have suppression ${ }^{32}$.

3. Accomplish this by the administration of a suppression test ${ }^{32}$. This is a crucial step because in the absence of normal binocularity, participants will not be able to recognize the fixation target and measurement of light source simultaneously and thus would confirm the appropriate location of measurement in the fovea and parafoveal regions.

4. Exclude all participants less than 18 years of age, with visual acuity worse than $20 / 40$, with retinal damage in the macula region (central part of the retina), glaucoma, diabetic retinopathy, hemorrhage, severe cataract, or vitreous opacities preventing ophthalmic imaging or MPR measurements.

5. Exclude participants who are unable to perform the measurements using heterochromatic flicker photometry or reflectometry, those for whom the devices are unable to provide MPOD values, or those with ocular suppression.

\section{Creating the peripheral track system (Figure 3)}

1. Obtain a slidable track with an approximately $1 \mathrm{~m}$ (3.5 feet) long aluminum rail that contains a hollow indent with space for a slidable track, such as a door weather strip. 
2. Mount the track $6.1 \mathrm{~m}$ (20 feet) from the subject seated at the MPR for the reflectometry measurements to be performed. Ensure that the track is $1.5 \mathrm{~m}$ off the ground to be at the same height as the participant's eye during the reflectometry measurement.

3. Mount three $1 \mathrm{~cm} \times 1 \mathrm{~cm}$ remote controlled LED lights on the slidable track so that the centers of the lights are spaced $10.7 \mathrm{~cm}$ away from each other.

NOTE: The $10.7 \mathrm{~cm}$ signify each degree and was determined because each LED light is $6.1 \mathrm{~m}$ away from the participant. The distance of $6.1 \mathrm{~m}$ ( $20 \mathrm{feet})$ is the minimum distance to obtain a true optical infinity, but if a track system is created at a further distance, the distance between each LED light would change and a new distance would have to be calculated trigonometrically. (See Table 1). If fewer than $6 \mathrm{~m}$ are utilized, pupillary dilation is advisable to minimize the ocular accommodation.

\section{Measurements using a heterochromatic flicker photometer}

NOTE: This step is for additional data collection and is not essential for peripheral measurements using the reflectometer.

1. Instill artificial tears in both eyes, ask the participant to blink a few times, and patch the eye that is not being tested.

2. Explain the procedure to the participant.

3. Instruct the participant to look at the central fixation target of the heterochromatic flicker photometer visible through the eyepiece and to press the clicker each time they observe the target flickering. Ensure that the fixation target flickers a total of five times to determine the initial threshold.

4. View the results of the initial threshold and remind the participant to click the button each time the central fixation target flickers as the test continues for $45 \mathrm{~s}$ to $1 \mathrm{~min}$.

5. A graph and MPOD value will appear on the control monitor along with a reliability index. Ensure that "acceptable" is displayed on the reliability index. Repeat the test if results indicate "borderline" or "unacceptable" until an "acceptable" reliability index is obtained.

6. Click on the next green arrow that appears on the control monitor once the participant has finished the test to save the results.

\section{Central measurement procedure using the reflectometer}

NOTE: The subsequent steps will lead to the measurement of individual carotenoids. This is performed using the reflectometer. The central measurements need not be performed to measure peripheral measurements with the reflectometer. However, the central measurements are important for clinical use.

1. Input the participant information into the reflectometer software.

2. Click the Run Eye Test tab.

3. White Calibration

NOTE: This is a pivotal step in the calibration of the spectrometer within the reflectometer device to full spectrum white sample. This is performed once a day when the device is turned on by the technician. A participant is not needed for this step.

1. Click the White button next to Calibrate.

2. Insert the white calibration tube onto the reflectometer after the message instructing the user to insert the "white calibration tube" is displayed on the screen.

3. Click OK once the white calibration tube is inserted to begin the white calibration. Ensure the Black button next to Calibrate has been enabled after the White Calibration Successful message has appeared on the screen.

4. Black Calibration

1. Instill a drop of artificial tears into the participant's eyes and have them place their chin onto the chin rest.

2. Instruct the participant to place their eye close to the eye cup. Using the joystick, gently position the system so the eye cup presses against the participant's eye socket and blocks the room light from the system.

3. Click the Black button to select Calibrate and align the system to the participant's pupil. Proper alignment is achieved when the pupil is centered in the circle displayed on the touch screen monitor.

4. Instruct the participant to adjust the rotating knob on the front of the system to obtain a clear target.

5. Click OK once the participant has properly adjusted the system to their vision. The system will automatically carry out a black calibration sequence. Once black calibration has been successfully completed, the Left Eye and Right Eye buttons will be enabled, and a Black Calibration Successful message will appear on the screen.

5. Start of measurement

1. Click the Left Eye or Right Eye button on the screen depending on which eye is being measured.

2. Ensure that the system displays the message Align System to Subject's Eye. Ensure that the system is aligned to the participant's pupil. Use the joystick to make fine adjustments.

3. Click the OK button on the screen to start the MPOD measurement. The measurement time is $30 \mathrm{~s}$. A minimum of $10 \mathrm{~s}$ is needed to obtain the parameters/results. A countdown timer will appear on the top of the screen displaying how much time is left for the measurement. Ask the participant to look at the fixation light and encourage them to only blink when necessary.

4. Use the joystick during the measurement to ensure the system stays in alignment with the participant's pupil.

5. Ensure the system displays a message stating Measurement Successful once the measurement is completed.

6. Click the OK button to finish.

7. Repeat steps $4.4-4.6 .6$ to test the other eye if needed. The entire process takes about 2-3 min. NOTE: To repeat the measurement on the same eye wait at least $30 \mathrm{~s}$ then repeat steps $4.6-4.6 .6$ 


\section{Peripheral measurement technique using reflectometer (Figure 3)}

NOTE: The untested eye will fixate on a target allowing for placement of the stimulus at various eccentricities from the fovea of the tested eye. This methodology requires normal binocularity to allow for correct positioning of the eye in which the macular pigment optical density is being measured.

1. Input participant information into the reflectometry software.

2. Click the Run Eye Test tab.

3. Peripheral track calibration

1. After the white and black calibration is performed, press the Left Eye or Right Eye button on the screen depending on which eye is to be measured.

2. The system will display a message Align System to the Subject's Eye. Ensure that the system is aligned to the participant's pupil. Use the joystick to make fine adjustments.

3. Turn on the LED light on the track system that is farthest right to the participant. At this time, the participant should be able to see both the light from inside the reflectometer with their right eye and the red LED light with their left.

4. Instruct the participant to direct the trained observer to adjust the peripheral track until they can superimpose both stimuli to the best of their ability.

NOTE: There can be variability between participants on how far their superimposed "calibration point" is due to anatomical differences.

4. Start of measurement

1. Turn off the LED light and switch on the next LED light (to the left) to carry out the next 1 degree eccentric measurement. Explain to the participant that they need to look at the new red LED light throughout the entire measurement.

2. Click the OK button on the screen to start the MPOD measurement. The measurement time is $30 \mathrm{~s}$. A countdown timer will appear on the top of the screen displaying how much time is left for the measurement. Ask the participant to look at the appropriate red LED light and encourage them to only blink when necessary.

3. Use the joystick during the measurement to ensure the system stays in alignment with the participant's pupil.

4. Ensure the system displays a message stating Measurement Successful once the measurement is completed.

5. Click the OK button to finish.

6. Repeat steps 5.3.1-5.4.5 to retake a measurement. NOTE: The entire process takes about 2-3 min. Two measurements are recommended for each degree to allow for the comparison. To repeat measurements at a different retinal eccentricity, change the degree separation in step 4.8 .

\section{Analysis (Figure 4)}

1. Make a copy of the file to be analyzed.

NOTE: The file analyzed was generated in steps 4.6 .6 and 5.4.5. This step is not essential but allows for various analyses performed without altering the original data.

2. Open the reflectometry software on the desktop.

3. Click Import on the left side of the application and choose the copied file to be opened.

4. Click Edit under the Subject Record tab. A new window will open. This will help obtain data from the desired time interval.

5. Move the bottom slide bar up from 0 to 10 to eliminate the first $10 \mathrm{~s}$ of measurement. NOTE: The slide bar should read 10-30. These slide bars can move up or down to choose the desired time interval to analyze. (see Figure 4).

6. Click the Exit button on the left side of this window. A warning window will pop up. Select OK to confirm the interval cuts.

7. Click Launch Analyzer on the bottom left of the program (see Table of Materials). A new window will open.

8. Click Best Fit at the bottom of the page. This will populate the first set of data, including L-OD and Z-OD.

9. Record the data.

10. Click Reset to select another analysis option.

11. Select Macular Pigment under the Receptor Model options.

12. Click Best Fit to populate the second set of data, including MPOD.

13. Click Save Solution to save this interval.

\section{Representative Results}

This study included eight participants ranging from 22-29 years of age. Table 1 describes how to calculate the distance to obtain each degree of eccentricity from the center of the macula. Table 2 provides the demographics of the participants. The study sample includes an equal number of males and females with a wide variety of ethno-racial diversity. Table 3 shows the mean results of MPOD obtained by both the devices and L-OD and Z-OD of all participants involved in the study at various eccentricities. The mean MPOD and standard deviation obtained by the heterochromatic flicker photometer and the reflectometry technique was 0.480 (SD 0.14) and 0.593 (SD 0.161 ) respectively. There was excellent correlation between the MPOD measurement obtained using the techniques with Person correlation coefficient $r=0.92(p<0.001)$. The Z-OD was larger centrally compared to the L-OD measured in the foveal region. The L-OD to Z-OD ratio centrally was $1: 2.61$. The Z-OD decreased as a function of eccentricity at the center of the fovea. At 1 degree from the central fovea the concentration of Z-OD measured by reflectometry decreased significantly, with an increase in L-OD. The L-OD to Z-OD ratio at 1 degree from central fixation was 1.38:1.0. In the parafoveal region at 2 degrees from central fixation the lutein became the predominant carotenoid and the L-OD to Z-OD ratio was 2.08:1.0. Tables 4, 5, and 6 show the data obtained from all eight subjects. Examining the tables, it is obvious that there is significant interindividual variability of L-OD, Z-OD, and MPOD values, indicating that the physiological limits of normality can be large. 


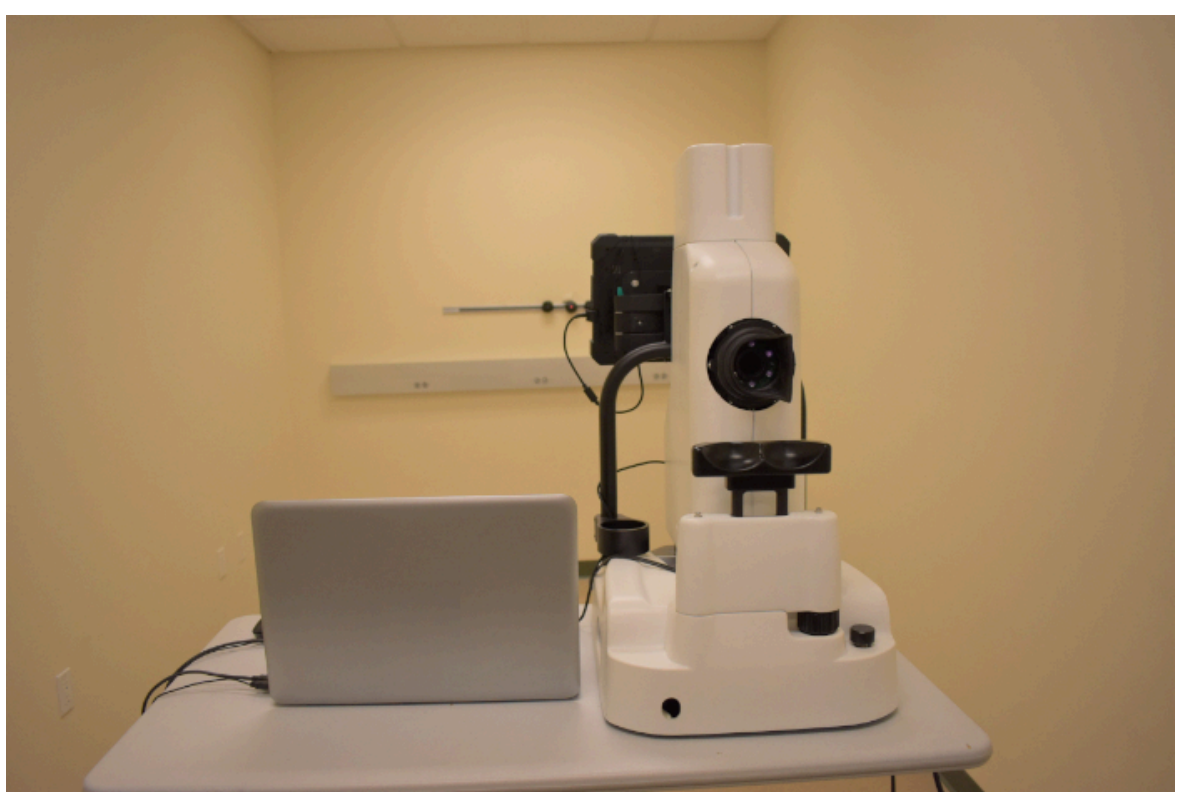

Figure 1: Macular pigment reflectometer. Macular Pigment Reflectometer used in this experiment. Please click here to view a larger version of this figure.

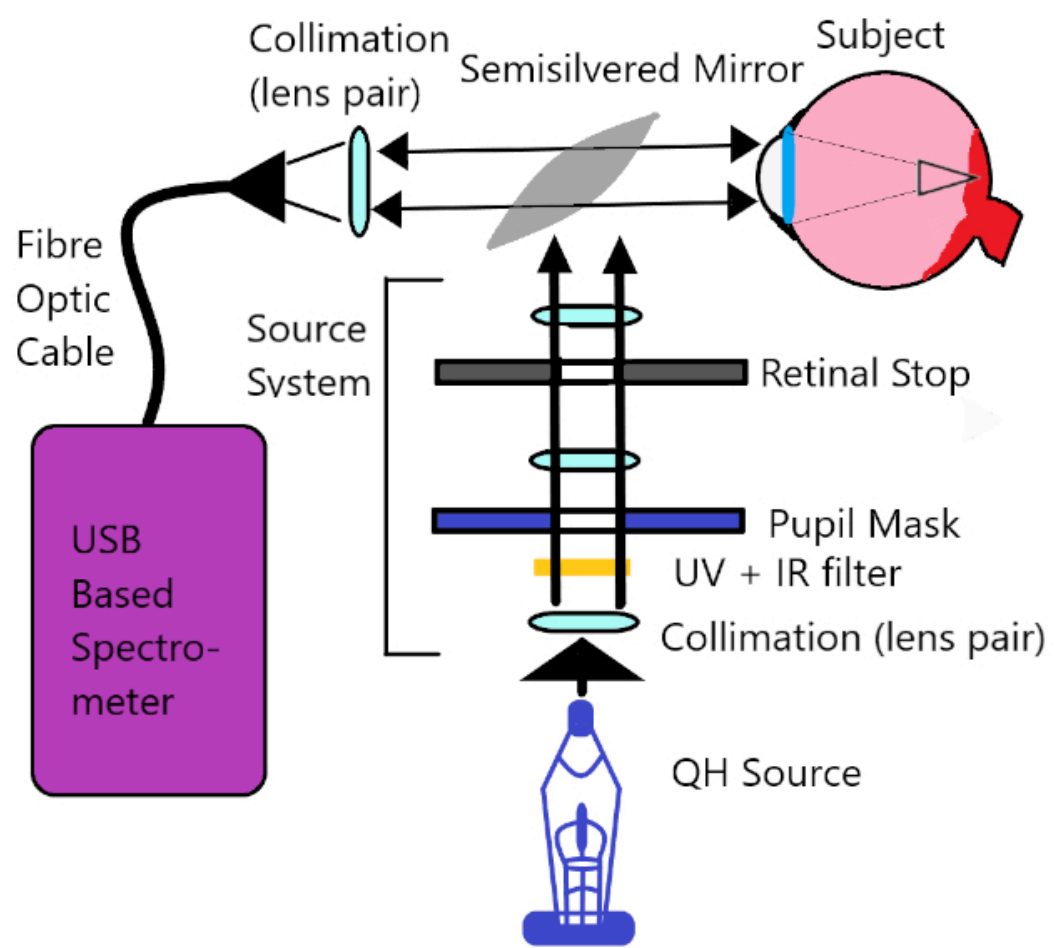

Figure 2: Macular pigment reflectometer operational schematic. Diagram of the internal operational schematics of the MPR device. Please click here to view a larger version of this figure. 


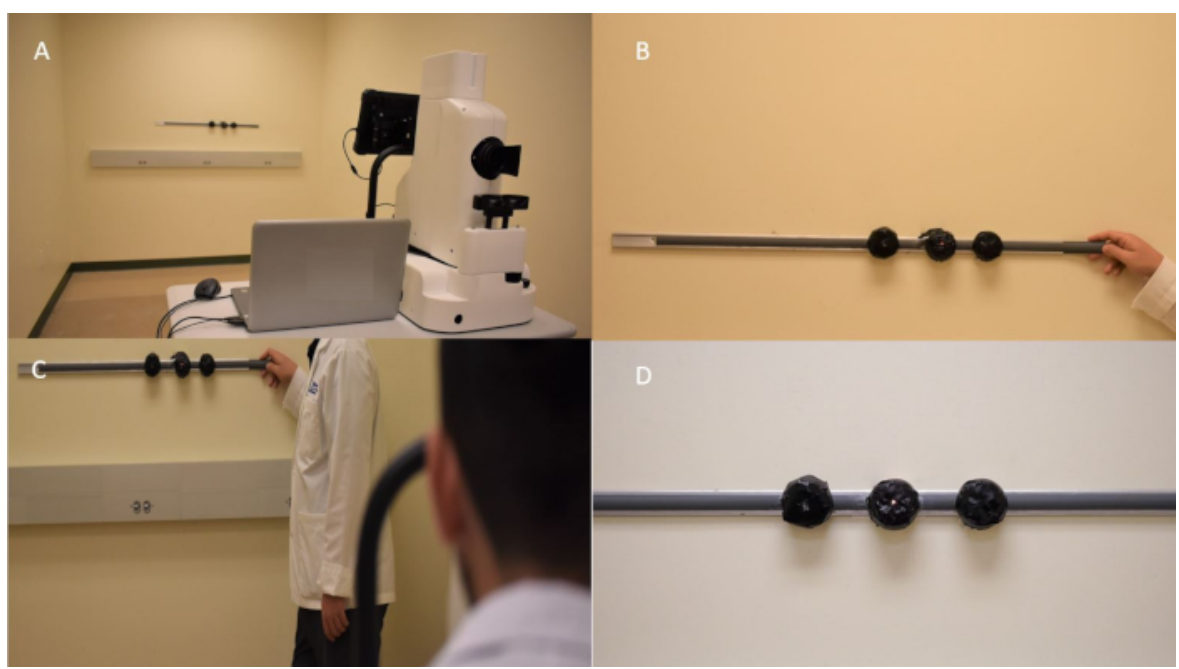

Figure 3: Peripheral measurement track system. (A) The macular pigment reflectometer with the peripheral track system $6.1 \mathrm{~m}$ away. (B) The track system with a researcher pointing at the 0 degree LED light. (C) The whole system as it would appear when the participant is being tested. (D) The track system with the 1 degree LED light on. Please click here to view a larger version of this figure.
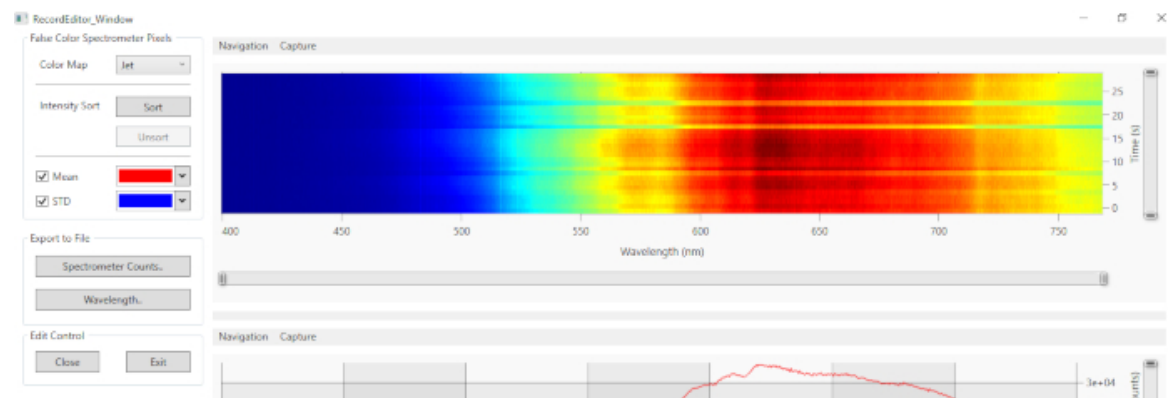

Figure 4: Window showing slide bars used for editing measurements to desired time. The slide bars used to edit the desired time frame. The image shows the first $10 \mathrm{~s}$ being removed. Please click here to view a larger version of this figure.

\begin{tabular}{|l|l|l|l|l|l|l|l|l|}
\hline $\begin{array}{l}\text { Test distance } \\
{[\mathrm{m}]}\end{array}$ & 3 & 4 & 5 & 6.1 & 7 & 8 & 9 & 10 \\
\hline $\begin{array}{l}\text { Distance } \\
\text { between } \\
\text { lights [m] }\end{array}$ & 0.052 & 0.07 & 0.087 & 0.107 & 0.122 & 0.14 & 0.157 & 0.175 \\
\hline
\end{tabular}

Table 1: Separation between fixation lights at various distances from target. The distance between the lights is the value for $x$ in this equation:

$\tan (1)=\frac{x}{d}$

where $d$ is the test distance.

\begin{tabular}{|c|c|c|c|c|}
\hline Subject & Age & Gender & Ethnicity & Race \\
\hline 3002 & 27 & $\mathrm{~F}$ & Hispanic & $\begin{array}{l}\text { Caucasian/More than one } \\
\text { race }\end{array}$ \\
\hline 3003 & 28 & $F$ & Hispanic & None \\
\hline 3004 & 26 & $F$ & Not Hispanic & African American \\
\hline 3005 & 24 & $M$ & Hispanic & Asian/More than one race \\
\hline 3006 & 27 & $M$ & Not Hispanic & Asian \\
\hline 3007 & 25 & $F$ & Not Hispanic & African American \\
\hline 3009 & 29 & $M$ & Hispanic & $\begin{array}{l}\text { Caucasian/More than one } \\
\text { race }\end{array}$ \\
\hline 3010 & 22 & $M$ & Not Hispanic & Asian \\
\hline
\end{tabular}


Table 2: Demographics of participants of the study. Table shows the age, gender, and ethnicity of the tested participants. The average age of the participants was 26 . There was a $1: 1$ ratio of men to women. The self-identified ethnicity of the participants included $50 \%$ Hispanic and about $37.5 \%$ of either Asian or More Than One Race.

\begin{tabular}{|l|l|l|l|l|l|}
\hline & Mean L-OD & Mean Z-OD & $\begin{array}{l}\text { Mean-reflectometry } \\
\text { MPOD }\end{array}$ & Mean Z-L Ratio & $\begin{array}{l}\text { Mean-Flicker } \\
\text { photomettry MPOD }\end{array}$ \\
\hline Central & 0.247 & 0.425 & 0.593 & $2.61: 1$ & 0.48 \\
\hline Peripheral 1 deg & 0.402 & 0.122 & 0.248 & $1: 1.38$ & Not available \\
\hline Peripheral 2 deg & 0.366 & 0.03 & 0.143 & $1: 2.08$ & Not available \\
\hline
\end{tabular}

Table 3: Mean values of carotenoids at various eccentricities. The table shows the mean results from the eight participants in the study. SD for mean central L-OD (0.188) and mean central Z-OD (0.142). SD for Mean Central MPOD of MPR (0.161) and SD for Mean Central MPOD of the reflectometer (0.14). SD for mean L-OD at peripheral 1 degree (0.224) and mean Z-OD at peripheral 1 degree (0.122). SD for mean MPOD of MPR at peripheral 1 degree (0.248). SD for mean L-OD at peripheral 2 degree $(0.366)$ and SD for mean Z-OD at peripheral 2 degree $(0.030)$. $\mathrm{SD}$ for mean MPOD of MPR at peripheral 2 degree $(0.143)$

\begin{tabular}{|c|c|c|c|c|c|}
\hline Participant & L-OD & Z-OD & MPOD & Z-L Ratio & MPS \\
\hline 3002 & 0.525 & 0.409 & 0.669 & 0.778 & 0.58 \\
\hline 3003 & 0.566 & 0.415 & 0.6525 & 0.733 & 0.48 \\
\hline 3004 & 0.1615 & 0.291 & 0.437 & 1.793 & 0.437 \\
\hline 3005 & 0.121 & 0.414 & 0.555 & 3.432 & 0.555 \\
\hline 3006 & 0.148 & 0.724 & 0.888 & 4.892 & 0.888 \\
\hline 3007 & 0.074 & 0.389 & 0.536 & 5.257 & 0.536 \\
\hline 3009 & 0.197 & 0.26 & 0.361 & 1.32 & 0.361 \\
\hline 3010 & 0.183 & 0.496 & 0.642 & 2.71 & 0.642 \\
\hline
\end{tabular}

Table 4: Individual carotenoid optical density measurements obtained at central fixation. Table shows the measurements obtained at central fixation for all eight participants.

\begin{tabular}{|l|l|l|l|l|}
\hline Participant & L-OD & Z-OD & MPOD & Z-L Ratio \\
\hline 3002 & 0.325 & 0 & 0.012 & 0 \\
\hline 3003 & 0.385 & 0.08 & 0.166 & 0.208 \\
\hline 3004 & 0.121 & 0.253 & 0.392 & 2.091 \\
\hline 3005 & 0.7015 & 0 & 0.119 & 0 \\
\hline 3006 & 0.362 & 0.286 & 0.45 & 0.79 \\
\hline 3007 & 0.104 & 0.265 & 0.391 & 2.548 \\
\hline 3009 & 0.589 & 0 & 0.183 & 0 \\
\hline 3010 & 0.626 & 0.094 & 0.273 & 0.15 \\
\hline
\end{tabular}

Table 5: Individual carotenoid optical density measurements obtained at 1 degree from central fixation. Table shows the measurements obtained at 1 degree from central fixation for all eight participants.

\begin{tabular}{|l|l|l|l|l|}
\hline Participant & L-OD & Z-OD & MPOD & Z-L Ratio \\
\hline 3002 & 0.146 & 0 & 0.043 & 0 \\
\hline 3003 & 0.351 & 0 & 0.066 & 0 \\
\hline 3004 & 0.063 & 0.077 & 0.169 & 1.222 \\
\hline 3005 & 0.189 & 0.017 & 0.067 & 0.09 \\
\hline 3006 & 0.902 & 0 & 0.291 & 0 \\
\hline 3007 & 0.04 & 0.099 & 0.201 & 2.475 \\
\hline 3009 & 0.718 & 0.046 & 0.232 & 0.064 \\
\hline 3010 & 0.518 & 0 & 0.076 & 0 \\
\hline
\end{tabular}

Table 6: Individual carotenoid optical density measurements obtained at $\mathbf{2}$ degrees from central fixation. Table shows the measurements obtained at 2 degrees from central fixation for all eight participants. 


\section{Discussion}

Our study illustrates the technique and methodology to perform in vivo MPOD measurements at various foveal and parafoveal regions using a reflectometer device. We developed and calibrated a peripheral track system to obtain measurements at 1 degree and 2 degrees from the central fixation. Our study results show that MPOD, L-OD, and Z-OD can be measured at various foveal and parafoveal regions using this protocol at optical infinity. The protocol could be adapted for shorter distances when lengthy rooms are not available in a clinic. In that case, however, pupillary dilation will be necessary to control active accommodation (see Table 1).

There are two critical steps when performing this experiment: 1) the 0 degree calibration and 2) the black calibration. When using the peripheral track system to measure MPOD and its constituents off-center, the external fixation for the 0 degree calibration or foveal measurement is of utmost importance. If the participant whose eye is measured does not understand this procedure or cannot perform the necessary steps, the measurements will be compromised and erroneous. The black calibration is also critical because it allows the MPR to establish a baseline spectrometer measurement when no light is present, which the device then compares to all values obtained from the subject. Therefore, the black calibration is a must for every participant.

Our study results indicate that the central MPOD levels match data from previous published experimental and histological studies ${ }^{7,10,14}$. Furthermore, we found that the levels for MPOD decline with increasing retinal eccentricity, with MPOD values being greater at the foveal compared to the parafoveal region. The lutein and zeaxanthin levels also vary at different retinal locations with the lutein and zeaxanthin ratios changing as a function of eccentricity. We found central L-OD and Z-OD ratios of 1:2.6, which changed to $2.08: 1$ at 2 degrees from central fixation. This is consistent with reports from previous studies ${ }^{10,29}$. We found that the lutein and zeaxanthin levels showed considerable interindividual variation. Prior in vivo laboratory experiments have evaluated only three subjects and there is limited information in this area ${ }^{29}$. If the significant interindividual variation of levels of carotenoids is correct, then this would support the need to obtain baseline measures of carotenoids and tailoring individual supplements. Further research will be needed to confirm this finding of high interindividual variability of lutein and zeaxanthin levels in healthy individuals. Prior publications and work with this MPR device show that repeatable measurements can be obtained for MPOD in both undilated and dilated pupillary conditions, although the repeatability of L-OD and Z-OD measurements was improved when pupils were dilated ${ }^{25}$. In the present study, we performed all MPR measurements with dilated pupils. Given that the carotenoid levels are lower at the foveal periphery and parafoveal region, it may be essential to dilate the pupil for consistent signal strength and reliable peripheral measurements.

We tried various methods, and ultimately developed and tested our track system. It proved to be the most effective way to achieve reliable results. The system was tested by examining three participants multiple times to see if similar outcomes could be achieved with each attempt. This included measuring the participants on three separate occasions over a two-month period. Other methods attempted included modifying the reflectometry eyepiece by creating a cover with precut slits at 0,1 , and 2 degrees off center. This technique proved ineffective because the slits were too close together for a subject to adequately distinguish.

There are several limitations in this study. The study requires that the subjects have normal binocularity. This ensures that the subject will be able to fixate on the target while the other eye is being measured. Subjects who do not meet this criterion will be unable to comply with the instructions, will not fixate properly while engaging the stimulus, and cannot be measured successfully using this technique. The track system was reliable, but its limitations could be addressed in future studies. The protocol could be improved by having built-in red LED fixation lights with part of a Badal optometer system as a part of the reflectometer. This would allow the participant to fixate at the desired eccentricity with the eye being measured with appropriate accommodation of lens.

At the present time, there are no alternative techniques to measure in vivo L-OD and Z-OD. However, alternative devices that measure MPOD exist. One such device is the heterochromatic flicker photometer used in this study. The heterochromatic flicker photometer employs a psychophysical method of testing and cannot determine individual lutein and zeaxanthin values. The central MPOD measurements obtained using a heterochromatic flicker photometer were an average of 0.11 lower than those obtained by the MPR device with a standard deviation of 0.16 . The MPOD measurement obtained using both the techniques had excellent correlation as reported previously ${ }^{25}$.

Although the current study has a small sample size, its purpose was to prove the concept that peripheral measurements of zeaxanthin and lutein optical density can be obtained using a reflectometry device. To our knowledge, other in vivo studies have had significantly smaller sample sizes than the sample utilized in this study. Therefore, we are confident that our results demonstrate that in vivo carotenoid density can be measured at the foveola, foveal periphery, and parafoveal region using a reflectometer. Our study sheds further light on how zeaxanthin and lutein levels are distributed in the central and peripheral macular regions within the human retina. Because we found a remarkable variation of the values among our study participants, larger studies both in vivo and in vitro are needed to better understand lutein and zeaxanthin distribution, levels, and ratios within the general population.

\section{Disclosures}

Dr. Pinakin Davey is a consultant for ZeaVision and Dr. Dennis Gierhart is an Employee, Chief Scientific Officer ZeaVision manufacturer of MPR device. Other authors report no conflicts.

\section{Acknowledgments}

We thank WesternU College of Optometry and the Master of Science in Medical Sciences program at WesternU for their assistance and support.

We also thank ZeaVision for their generous support and funding. 


\section{References}

1. Handelman, G. J., Dratz E. A., Reay, C. C., van Kuijk, F. Carotenoids in the human macula and whole retina. Investigative Ophthalmology \& Visual Science. 29 (6), 850-855 (1988).

2. Milani, A., Basirnejad, M., Shahbazi, S., Bolhassani, A. Carotenoids: biochemistry, pharmacology and treatment. British Journal of Pharmacology. 174 (11), 1290-1324 (2017).

3. Bhosale, P., Zhao, D. Y., Bernstein, P. S. HPLC measurement of ocular carotenoid levels in human donor eyes in the lutein supplementation era. Investigative Ophthalmology \& Visual Science. 48 (2), 543-549 (2007).

4. Zimmer, J. P., Hammond B.R. Possible influence of lutein and zeaxanthin on the developing retina. Clinical Ophthalmology. 1 (1), 25-35 (2007).

5. Friedman, D. S. et al. Prevalence of Age-Related Macular Degeneration in the United States. Archives of Ophthalmology. 122 (4), 564-572 (2004).

6. Ambati, J., Fowler, B. J. Mechanisms of age-related macular degeneration. Neuron. 75 (1), $26-39$ (2012).

7. Bernstein, P. S., Delori, F. C., Richer, S., van Kuijk, F. J., Wenzel, A. J. The value of measurement of macular carotenoid pigment optical densities and distributions in age-related macular degeneration and other retinal disorders. Vision Research. 50 (7), $716-728$ (2010).

8. Bone, R. A., Landrump, J.T., Hime, G. W., Cains, A., Zamor, J. Stereochemistry of the Human Macular Carotenoids. Investigative Ophthalmology \& Visual Science. 34 (6), 2033-2040 (1993).

9. Leung, I. Y. Macular pigment: New clinical methods of detection and the role of carotenoids in age-related macular degeneration. Optometry Journal of the American Optometric Association. 79 (5), 266-272 (2008).

10. Bone, R. A. et al. Distribution of Lutein and Zeaxanthin Stereoisomers in the Human Retina. Experimental Eye Research. 64 (2), $211-218$ (1997).

11. de Kinkelder, R. et al. Macular pigment optical density measurements: evaluation of a device using heterochromatic flicker photometry. Eye. 25 (1), 105-112 (2011).

12. Snodderly, D. M., Auran, J. D., Delori, F. C. The macular pigment. II. Spatial distribution in primate retinas. Investigative Ophthalmology \& Visual Science. 25 (6), 674-685 (1984).

13. Snodderly, D. M., Brown, P. K., Delori, F. C., Auran, J. D. The macular pigment. I. Absorbance spectra, localization, and discrimination from other yellow pigments in primate retinas. Investigative Ophthalmology \& Visual Science. 25 (6), 660-673 (1984).

14. Bone, R. A., Landrum, J. T., Fernandez, L., Tarsis, S. L. Analysis of the Macular Pigment by HPLC: Retinal Distribution and Age Study. Investigative Ophthalmology \& Visual Science. 29 (6), 843-849 (1988).

15. Chous A. P., Richer S. P., Gerson, J. D., Kowluru, R. A. The Diabetes Visual Function Supplement Study. British Journal of Ophthalmology. 100 (2), 227-234 (2016).

16. Vishwanathan, R., Schalch, W., Johnson, E. J. Macular pigment carotenoids in the retina and occipital cortex are related in humans. Nutritional Neuroscience. 19 (3), 95-101 (2016).

17. Barnett, S. M. et al. Macular pigment optical density is positively associated with academic performance among preadolescent children, Nutritional Neuroscience. 21 (9), 632-640 (2018).

18. Saint, S. E. et al. The Macular Carotenoids are Associated with Cognitive Function in Preadolescent Children. Nutrients. 10 (2), 193 (2018).

19. Johnson, E. J. et al. Relationship between Serum and Brain Carotenoids, $\alpha$-Tocopherol, and Retinol Concentrations and Cognitive Performance in the Oldest Old from the Georgia Centenarian Study. Journal of Aging Research. 2013, 951786 (2013).

20. Hammond, B. R. et al. Effects of Lutein/Zeaxanthin Supplementation on the Cognitive Function of Community Dwelling Older Adults: A Randomized, Double-Masked, Placebo-Controlled Trial Front. Aging Neuroscience. 3 (9), 254 (2017).

21. Renzi-Hammond, L. M., et al. Effects of a Lutein and Zeaxanthin Intervention on Cognitive Function: A Randomized, Double-Masked, Placebo-Controlled Trial of Younger Healthy Adults. Nutrients. 9 (11), 1246 (2017).

22. Wooten, B. R., Hammond, B. R. Spectral Absorbance and Spatial Distribution of Macular Pigment Using Heterochromatic Flicker Photometry. Optometry and Vision Science. 82 (5), 378-386 (2005).

23. Putnam, C. M. Clinical imaging of macular pigment optical density and spatial distribution. Clinical and Experimental Optometry. 100 (4), 333-340 (2017).

24. Davey, P. G., Alvarez, S. D., Lee, J. Y. Macular pigment optical density: repeatability, intereye correlation, and effect of ocular dominance. Clinical Ophthalmology. 10, 1671-1678 (2016).

25. Davey, P. G., Ngo, A., Cross, J., Gierhart, D. L. Macular pigment reflectometry: development and evaluation of a novel clinical device for rapid objective assessment of the macular carotenoids. Proceedings of SPIE 10858, Ophthalmic Technologies XXIX. 1085828 (2019).

26. Rapp, L. M., Maple, S. S., Choi, J. H. Lutein and Zeaxanthin Concentrations in Rod Outer Segment Membranes from Perifoveal and Peripheral Human Retina. Investigative Ophthalmology \& Visual Science. 41 (5), 1200-1209 (2000).

27. van de Kraats, J., Berendschot, T. T., Valen, S., van Norren, D. Fast assessment of the central macular pigment density with natural pupil using the macular pigment reflectometer. Journal of Biomedical Optics. 11 (6), 064031 (2006).

28. Sommerburg, O. et al. Lutein and zeaxanthin are associated with photoreceptors in the human retina. Current Eye Research. 19 (6), $491-495$ (1999).

29. van de Kraats, J., Kanis, M.J., Genders, S.W., van Norren, D. Lutein and zeaxanthin measured separately in the living human retina with fundus reflectometry. Investigative Ophthalmology \& Visual Science. 49 (12), 5568-5573 (2008).

30. van der Veen, R.L.P. et al. A new desktop instrument for measuring macular pigment optical density based on a novel technique for setting flicker thresholds. Ophthalmic and Physiological Optics. 29 (2), 127-137 (2009).

31. Howells, O., Eperjesi, F., Bartlett, H. Measuring macular pigment optical density in vivo: a review of techniques. Graefe's Archive for Clinical Experimental Ophthalmology. 249 (3), 315-347 (2011).

32. Howells, O., Eperjesi, F., Bartlett, H. Improving the repeatability of heterochromatic flicker photometry for measurement of macular pigment optical density. Graefe's Archive for Clinical and Experimental Ophthalmology. 251 (3), 871-880 (2013). 